\title{
Interleukin 8 is a biomarker of telomerase inhibition in cancer cells
}

\author{
Peter Solomon', Yuying Dong ${ }^{1}$, Shaillay Dogra ${ }^{2}$ and Romi Gupta ${ }^{1 *}$
}

\begin{abstract}
Background: Telomerase activity is required for both initiation and maintenance of tumorigenesis and over 90\% cancers overexpress telomerase. Therefore, telomerase targeting has emerged as a potential strategy for cancer treatment. In agreement with this, several telomerase inhibitors are being tested for cancer treatment and have shown some promise. However, because of the variability in response between the cancer patients, it is important to identify biomarkers that allow for distinguishing cancers that are responsive to telomerase inhibition from the cancers that are not. Therefore, in this study we performed experiments to identify a biomarker that can be used to predict telomerase inhibition induced tumor growth inhibition.
\end{abstract}

Methods: In our study, we have performed transcriptome-wide gene expression analysis on multiple ovarian and colon cancer cell lines that were treated with telomerase inhibitor imetelstat and were responsive to telomerase inhibition-induced tumor growth attenuation.

Results: We demonstrate that telomerase inhibition by telomerase inhibitor imetelstat results in decreased expression of interleukin 8 (IL8) in all telomerase responsive cancer cell lines. This phenomenon is of general occurrence because we find that multiple ovarian and colon cell lines show decrease in IL8 mRNA and protein levels after telomerase inhibition. Additionally, we find loss of IL8 phenocopy Telomerase inhibition mediated growth inhibitory effect in cancer cells.

Conclusion: Taken together, our results show that IL8 is a biomarker that predict telomerase inhibition mediated growth attenuation of cancer cells and its loss phenocopy telomerase inhibition. Therefore, IL8 expression can be utilized as a biomarker for telomerase targeted cancer therapies to potentially predict therapeutic response.

Keywords: Telomerase, Interleukin, Viability, Biomarker, Response

\section{Background}

Telomeres are region of repetitive nucleotide sequences at the end of a chromosome that protects chromosomal ends from fusion or from being recognized as damaged DNA [1-3]. In normal cells, during every DNA replication cycle, the telomere ends become shorter due to the end replication problem, which consequentially results in replicative senescence [4-6]. Telomerase is an RNA-protein enzyme complex responsible for maintaining telomere length and telomerase activity is required for both initiation and maintenance of human cancers [7-9]. Telomerase is activated in approximately $90 \%$ of tumors and is an important event in cellular immortalization process $[8,10,11]$. The mechanisms of telomerase overexpression in cancer cells

\footnotetext{
* Correspondence: Romi.Gupta@yale.edu

${ }^{1}$ Department of Pathology, Yale University School of Medicine, LH-306, New Haven, CT 06510, USA

Full list of author information is available at the end of the article
}

are still not clear. Some studies propose that telomerase promoter can acquire mutations that result in generation of new transcription factor binding sites and activation of telomerase [12]. Furthermore, since over $90 \%$ of cancer types express telomerase, telomerase represents an important target for cancer therapy [13-15]. However, telomerase targeting-based cancer therapies have thus far provided only limited success [16-19], which highlights the importance of identifying the biomarker that might predict the response to telomerase inhibition.

In our previous study, we have shown that simultaneous inhibition of CDKN1A and telomerase by imetelstat (JNJ-63935937, also known as GRN163L) leads to synergistic tumor growth inhibition [20]. In this study, we aimed to identify the biomarker that can predict response to telomerase inhibition-based therapy. To do so, we performed transcriptome-wide gene expression

(c) The Author(s). 2018 Open Access This article is distributed under the terms of the Creative Commons Attribution 4.0 International License (http://creativecommons.org/licenses/by/4.0/), which permits unrestricted use, distribution, and reproduction in any medium, provided you give appropriate credit to the original author(s) and the source, provide a link to the Creative Commons license, and indicate if changes were made. The Creative Commons Public Domain Dedication waiver (http://creativecommons.org/publicdomain/zero/1.0/) applies to the data made available in this article, unless otherwise stated. 
analysis on multiple ovarian and colon cancer cell lines that were treated with telomerase inhibitor imetelstat and were responsive to telomerase inhibition-induced tumor growth attenuation. Imetelstat is a potent and specific telomerase inhibitor and in clinical trials for cancer treatment $[21,22]$. We find that upon treatment with telomerase inhibitor imetelstat, expression of IL8 mRNA and protein is inhibited in multiple ovarian and colon cancer cells. We also show that IL8 inhibition also suppresses the cancer cell viability. These results indicate that inhibition of IL8 upon telomerase inhibition by imetelstat act as biomarker to predict the response to telomerase-based therapy.

\section{Methods}

\section{Cell culture}

HCT116 cells were a kind gift of Bert Vogelstein (Johns Hopkins Medical School). COLO205 (ATCC ${ }^{\circledast}$ CCL-222 $2^{\mathrm{TM}}$ ) and OVCAR5 cells (obtained as part of NCI-60 cell line panel, NCI) were obtained from American Type Culture Collection (ATCC) and were grown as recommended. Cells were treated with $2.5 \mu \mathrm{M}$ imetelstat or mismatch oligonucleotide (Geron Corporation) twice per week for up to 6 weeks and did not exceed $80 \%$ confluence during the treatment. IL8 (GFP tagged) cDNA ORF was obtained from origene. Reagents RPMI 1640 (Catalog no. \# 11879020, Thermofischer scientific), DMEM (Catalog no. \# 11966092, Thermofischer scientific), Penicillin-streptomycin (Catalog no. \# 15140122, Thermofischer scientific) and Fetal Bovine serum (Catalog no. \# 10082147, Thermofischer scientific) were purchased from Thermofischer scientific.

\section{Transfections, shRNAs and preparation of lentiviral particles}

IL8 and control non-specific shRNAs were obtained from Open Biosystems. Table 1 shows the product IDs for all shRNAs. Lentiviral particles were prepared by co-transfecting the shRNA plasmids and lentiviral packaging plasmids, pSPAX2 and pMD2.G, into $293 \mathrm{~T}$ cells using Effectene (Qiagen) and following the protocol at the Broad Institute's website (http://www.broadinstitute.org/rnai/public/resources/protocols).

\section{Cell viability, Colony formation assay and tumorigenesis assays}

To measure cell viability two kinds of assays were performed. For Trypan blue exclusion viability assays, cells were plated and were treated with mismatch oligo's or imetelstat for 2 weeks triplicate. After 2 weeks of treatment they were trypsinized and were with mixed with an equal volume of Trypan Blue Solution (Invitrogen) and counted using Countess (Invitrogen). Relative cell viability of imetelstat treated cells with respect to control mismatch oligo treated cells is plotted. For MTT assay cells are plated in in 96 well plate. Then they were either treated with mismatch oligo's or imetelstat for 2 weeks. After treatment, $10 \mu \mathrm{l}$ of $5 \mathrm{mg} / \mathrm{ml}$ of MTT is added to the medium each well and incubated for $1 \mathrm{~h}$. Medium is removed and $100 \mu \mathrm{l}$ of DMSO is added, mixed well and the measurement is performed at absorbance 590 and $630 \mathrm{~nm}$ wavelength. Average is taken and the reading at 690 is subtracted form that at 590 . Relative cell viability of imetelstat treated cells with respect to control mismatch oligo treated cells is plotted. For colony formation assays, $10^{3}$ cells were seeded in triplicate. Then they were either treated with mismatch oligo's or imetelstat for 2 weeks. Colonies formed were stained with $0.005 \%$ crystal-violet solution and counted. For in vivo experiment, Eight-week old, athymic nude ( $\mathrm{NCr}$ nu/nu) mice $(n=5)$ were injected subcutaneously with cancer cells $\left(2.5 \times 10^{6}\right)$. After one week, tumor-bearing mice received mismatch oligonucleotide or imetelstat $(30 \mathrm{mg} / \mathrm{kg}$ bodyweight) three times per week by intraperitoneal injection. Tumor growth was measured every week using calipers, and tumor volumes were calculated using the formula $0.5 \mathrm{X}$ length $\mathrm{X}$ width $^{2}$ for every week.

\section{Quantitative RT-PCR and immunoblot analysis}

qRT-PCR and Immunoblot was performed as described in [20]. Briefly For mRNA expression analyses, total RNA was extracted with TRIzol (Invitrogen) and purified using RNAeasy mini columns (Qiagen), and cDNA was generated using M-MuLV first-strand cDNA synthesis kit (New England Biolabs) as per manufacturer's instructions. Quantitative RT-PCR was performed using Power SYBR-green kit (Applied Biosystems) for mRNA expression analysis or the miScript SYBR-green PCR assay kit (Qiagen), as per manufacturer's instructions. Actin was used as an internal control. For Immunoblotting whole cell protein extracts were prepared using IP lysis buffer (Pierce) containing Protease Inhibitor Cocktail and Phosphatase Inhibitor Cocktail (Sigma-Aldrich, St.Louis, $\mathrm{MO})$. Protein concentration was estimated using a Bradford Assay kit (Bio-Rad). Proteins separated on 10\% or $12 \%$ polyacrylamide gels were transferred to PVDF membranes using a wet transfer apparatus from Biorad. Membranes were blocked with 5\% skim milk and probed with primary antibodies followed by the appropriate secondary HRP-conjugated antibody (GE healthcare, UK). Blots were developed using the Supersignal Pico Reagent. Antibody and primer information is provided in Table 1.

\section{TRAP assay and telomere length measurement}

The TRAP assay was performed essentially as described [23]. Every plate included standards, inactivated samples and lysis buffer as controls. Each sample was analyzed at least in triplicate. Telomerase activity was plotted 
Table 1 Primer sequences for RT-qPCR analysis; clone ID and catalog numbers for shRNAs (Open Biosystems); antibodies used

\begin{tabular}{llll}
\hline Application & Gene symbol & Forward primer (5'-3') & Reverse primer (5'-3') \\
\hline RT-qPCR & IL8 & taaaaagccaccggagcact & atcaggaaggctgccaagag \\
& actin & gccgggacctgactgactac & Cattctccagggaggagctog number \\
shRNAs & Gene symbol & Clone ID & RHS3979-9625212 \\
& IL8 & TRCN0000058028 & RHS3979-9625214 \\
Antibodies & Protein & TRCN0000058030 & Dilution \\
& IL8 & Source & $1: 500$ dilution \\
& GFP & Santa Cruz Biotechnology & $1: 500$ dilution \\
Inhibitor & Actin & Santa Cruz Biotechnology & $1: 1000$ dilution \\
Imetelstat & Concentration & Cell Signaling & Source \\
Mismatch Oligonucleotide & $2.5 \mu \mathrm{M}$ & Geron Corporation & Geron Corporation \\
\hline
\end{tabular}

relative to control mismatch oligonucleotide treated cells. Telomere length measurement was performed using Relative Human Telomere length Quantification qPCR assay kit from Science cell (Catalog no. \# 8908). The assay was performed as described by the supplier.

\section{Transcriptome-wide gene expression measurement assay and data analysis}

For microarray experiments using HCT116, OVCAR5 and COLO205 (5 million) cells were treated with imetelstat for 2 weeks, total RNA was isolated from the cells as described above and used to generate labelled antisense RNA. All antisense RNAs were made using the Ambion MessageAmp Kit and hybridized to Illumina HumanHT-12 V4.0 expression BeadChip using Illumina's protocol.

The microarray data were processed using GenomeStudio $^{\text {тx }}$ (Illumina), log2-transformed, and quantile-normalized using the "lumi" package of Bioconductor. All samples passed quality-control (QC) assessment, which included checking various control plots as suggested by Illumina, as well as other standard microarray-related analyses. Differential expression analyses were performed using the "limma" package, and a moderated $\mathrm{t}$-test with a Benjamini-Hochberg multiple testing correction procedure was used to determine statistical significance (adjusted $P$-value, $<0.05)$. Pathway analysis of differentially expressed genes for each comparison was performed using MetaCore $^{\mathrm{mu}}$ (version 6.8 build 29,806, GeneGo). Microarray data were submitted to Gene Expression Omnibus. Geo accession number is GSE106539.

\section{Statistical analysis}

All the experiments were conducted in three biological replicates. The results for individual experiments were expressed as mean \pm SEM. The $p$-values were calculated using t-test by using GraphPad Prism version $6.0 \mathrm{~h}$ for
Macintosh, GraphPad Software, San Diego California USA (www.graphpad.com).

\section{Results}

Identification of telomerase inhibition responsive Cancer cell lines

To identify biomarker of telomerase-inhibitor therapy, we analyzed a series of cancer cell lines of different tissue origin (Table 2). To this end, we treated these cancer cell lines, with imetelstat, a telomerase inhibitor. Imetelstat is a synthetic lipid-conjugated, 13-mer oligonucleotide N3' P5'-thio-phosphoramidate and is complementary to the template region of telomerase RNA (hTR). Inside the cells, imetelstat acts as a competitive enzyme inhibitor that binds and blocks the active site of the enzyme (a "telomerase template antagonist") thus inducing growth inhibition $[24,25]$.

We first optimized the imetelstat concentration for the treatment of various cancer cell lines. To do so, we treated different cancer cell lines with multiple concentrations of imetelstat for determining IC50. We found that IC50 for imetelstat for different cancer cell lines was in the range of $2.5 \mu \mathrm{M}$ as shown in Additional file 1: Figure S1. Next, we treated multiple ovarian and colon

Table 2 Cell lines and their tissue origin

\begin{tabular}{lll}
\hline S. No. & Cell lines & Tissue Origin \\
\hline 1 & SW480 & Colon \\
2 & KM12 & Colon \\
3 & HCT116 & Colon \\
4 & COLO205 & Colon \\
5 & OVCAR3 & Ovary \\
6 & ADR-RES & Ovary \\
7 & SKOV3 & Ovary \\
8 & OVCAR5 & Ovary \\
\hline
\end{tabular}



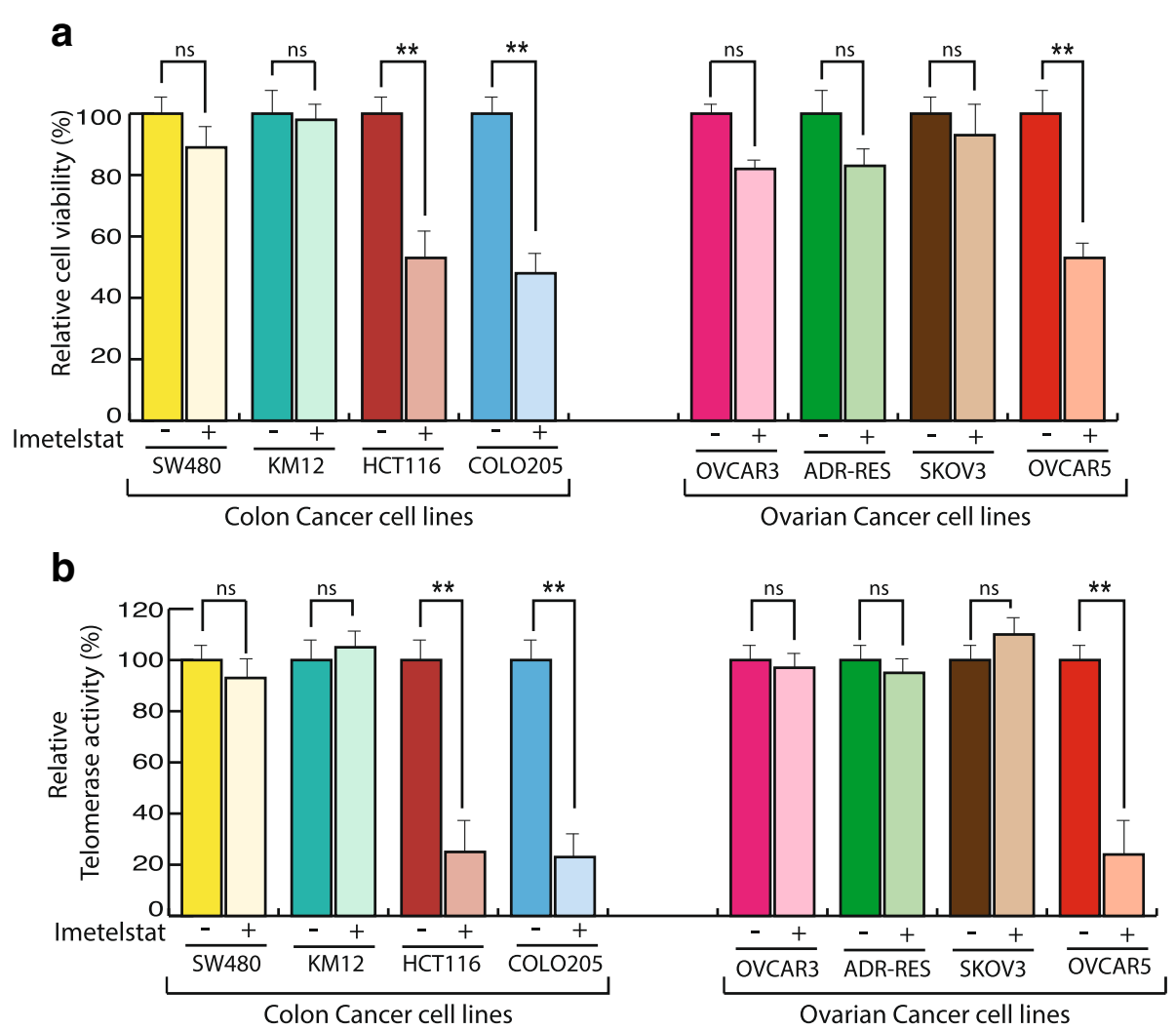

Fig. 1 Measurement of response of cancer cells to telomerase inhibition. Indicated cell lines were treated with mismatch oligonucleotide nucleotide or imetelstat for 2 weeks. a Cell viability was monitored by trypan blue exclusion assay and relative cell viability with respect to mismatch oligonucleotide nucleotide treated cell is plotted (b) Telomerase activities was measured by TRAP assay and relative telomerase activity with respect to mismatch oligonucleotide nucleotide treated cell is plotted. Error bar shows Standard Error Mean (SEM). (**, $p<0.001$ ), (ns, non-significant)
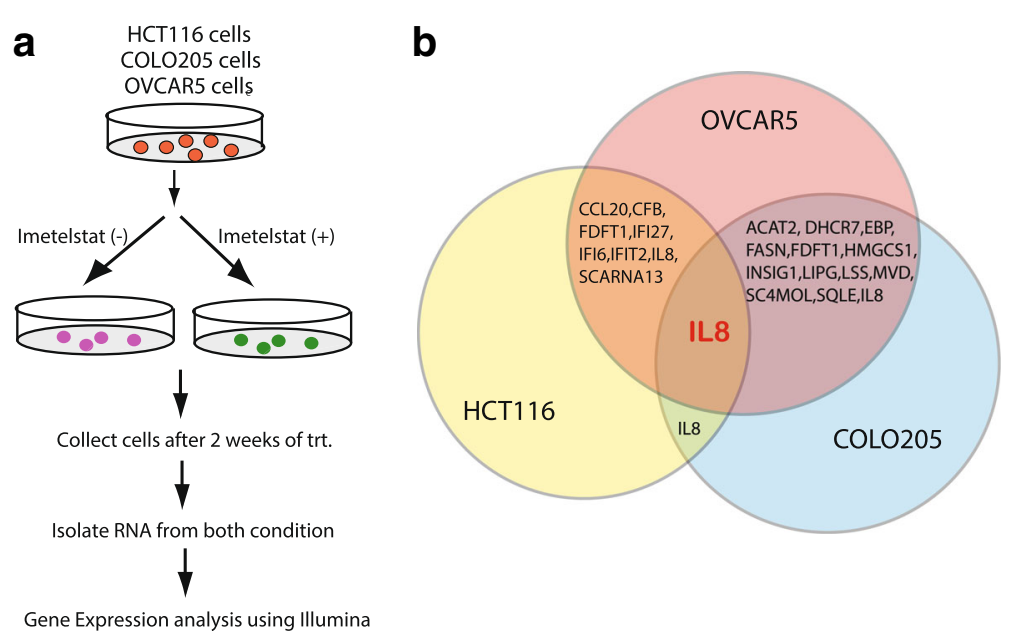

Fig. 2 Gene expression analysis using Illumina identified IL8 as a common biomarker of telomerase response. a Schematics of the Transcriptomewide gene expression analysis is described. It was performed in HCT116, OVCAR5 and COLO205 cells. Briefly equal number of cells from these cell lines were either untreated or treated with imetelstat for 2 weeks after which RNA was isolated and candidate genes that were either upregulated or downregulated were identified. $\mathbf{b}$ Venn diagram showing the common genes that were identified after imetelstat treatment in between two cell lines and also between three cell lines 
cancer cell lines of different tissue origin for 2 weeks with $2.5 \mu \mathrm{M}$ imetelstat and identified HCT116, COLO205 and OVCAR5 as three cancer cell lines that showed strong growth inhibition following imetelstat treatment and also showed concomitant decrease in telomerase activity (Fig. 1). We additionally checked telomere length in the cell lines that showed growth inhibition upon imetelstat treatment and found that HCT116, COLO205 and OVCAR5 show shortened telomere length upon imetelstat treatment as compared to control treated cells (see Additional file 2: Figure S2). These results demonstrate that inhibition of telomerase in ovarian and colon cancer cell lines leads to their growth attenuation.

Transcriptome-wide gene expression analysis identifies IL8 as a biomarker that predict response to telomerase inhibition

In order to identify the genes that can function as biomarker to predict response to telomerase-based therapy, HCT116, COLO205 and OVCAR5 cell lines that showed strong growth inhibition following imetelstat treatment were treated with imetelstat for 2 weeks and then transcriptome-wide gene expression analysis was performed (Fig. 2a). We observed multiple genes that were upregulated and downregulated in each cancer cell line (Fig. 2b). We also performed analysis to identify the common genes that were altered upon imetelstat treatment in all these cancer cell lines. Our results showed that in all three cancer cell lines, IL8 was the only common candidate that was downregulated in imetelstat treated cells as compared to control cells (Fig. 2b). These results in sum indicate that the cancer cells that show response to telomerase inhibition downregulate IL8 levels and this phenomenon is of general occurrence as multiple ovarian and colon cancer cell lines that undergo growth inhibition upon imetelstat shows IL8 downregulation (Fig. 2b).

\section{Telomerase inhibition suppresses cancer cell viability}

In Fig. 1, we have shown the effect of imetelstat on cancer cell viability using trypan blue exclusion assay. In order to confirm the growth inhibitory effect of imetelstat on HCT116, COLO205 and OVCAR5 cells we employed an additional assay called MTT assay. MTT is a calorimetric based assay to detect metabolic activity of cell which is dependent of the number of viable cells. To do so, we treated HCT116, COLO205 and OVCAR5

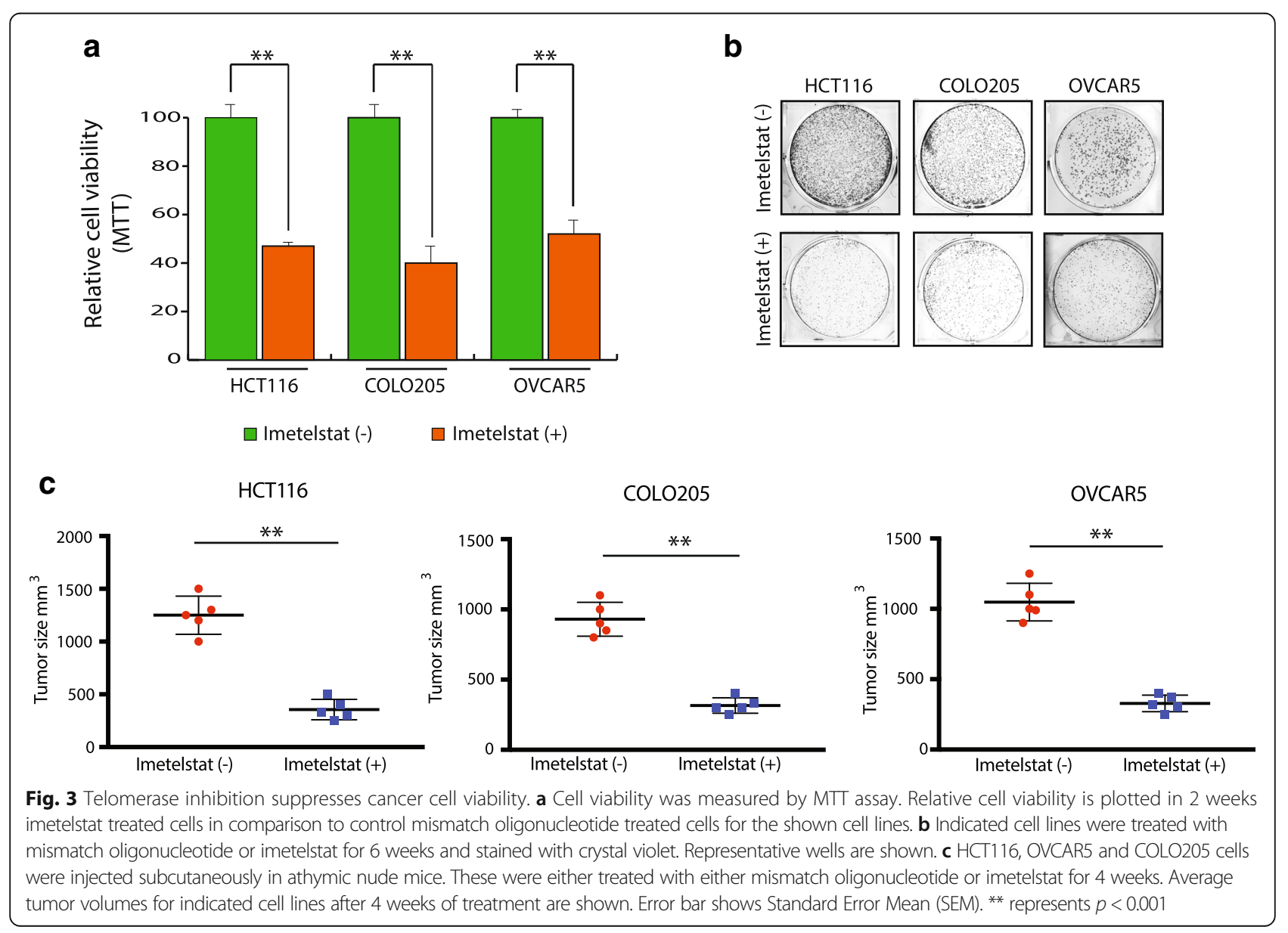




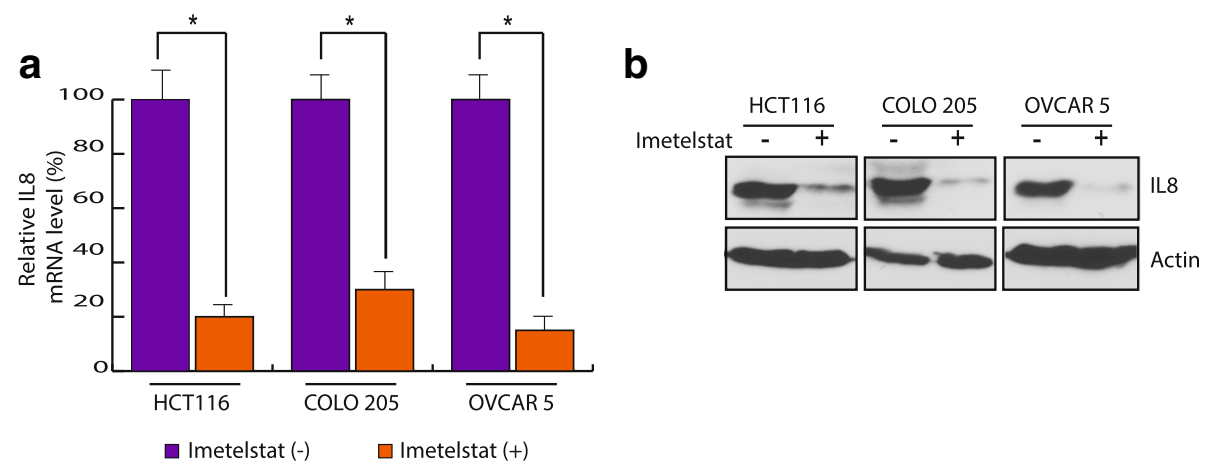

Fig. 4 IL8 levels decrease upon inhibition of telomerase. Indicated cells were treated with imetelstat for 2 weeks. a IL8 RNA level was measured by qRT-PCR. Actin was used as internal control. Relative levels with respect to control mismatch oligonucleotide treated cell is plotted. $\mathbf{b} I L 8$ protein level was measured by immunoblotting. Actin was used as loading control. Error bar shows Standard Error Mean (SEM). $\left(^{*}, p<0.01\right)$

cells with imetelstat for 2 weeks and then performed MTT assay as described in method section. As expected, we found that after two weeks of imetelstat treatment HCT116, COLO205 and OVCAR5 cells showed significant growth inhibition (Fig. 3a). This was further confirmed by colony formation assay as shown in Fig. 3b. Next, in order to study the effect of imetelstat on the growth of these cancer cell lines in vivo, we performed xenograft-based mouse tumorigenesis assay. Our results showed that imetelstat significantly inhibited the growth of HCT116, COLO205 and OVCAR5 tumors upon imetelstat treatment (Fig. 3c). These results confirmed that the inhibition of telomerase affects the cancer cell growth. Therefore, expression of telomerase is critical for cancer cell survival and serves as an effective target to inhibit cancer cell growth.

\section{Telomerase inhibition decreases both IL8 MRNA and protein level}

After confirming the cancer cells growth suppression upon telomerase inhibition, we measured IL8 transcript and protein level in HCT116, COLO205 and OVCAR5 cells after imetelstat treatment and found that both IL8 mRNA as well as IL8 protein level decreases upon imetelstat treatment (Fig. 4). This was observed in multiple ovarian and colon cancer cell lines suggesting that decrease in IL8 level is a common biomarker predictive of telomerase inhibition induced cancer cells growth suppression (Fig. 4). We also checked IL8 levels in the cell lines that did not show growth suppression upon telomerase inhibition and found that in these cell lines IL8 level did not change upon imetelstat treatment, as shown in Additional file 3: Figure S3. These results in sum indicate that decrease in IL8 level is a specific biomarker for the cancer cell lines that shown growth suppression upon telomerase inhibition.
IL8 inhibition affects the cell viability and its overexpression rescues telomerase inhibition induced growth inhibitory effect

Our result showed that IL8 level decreases upon imetelstat treatment. To confirm if the decrease in IL8 levels leads to decrease in cell viability, we knocked down IL8 expression using shRNAs in different cancer cell lines (Fig. 5a). Next, these cells were checked for cell viability. As shown in Fig. 5b, we find that the cells expressing IL8 shRNA have lower cell viability as compare to control cells expressing non-specific shRNA. In order to conclusively show the inhibition of IL8 is involved in telomerase inhibition induced growth inhibitory effect, we overexpressed IL8 in imetelstat treated cells (Fig. 5c), and then checked the cell viability. We found that IL8 overexpression rescued telomerase inhibition induced growth inhibitory effect (Fig. 5d). This was not due to restoration of telomerase activity upon IL8 expression, because no change in telomerase activity was observed after IL8 over expression in imetelstat treated cells (Fig. 5e). Taken together, these results led us to conclude that telomerase inhibition leads to decreases IL8 levels, which can be employed as a biomarker for predicting response to telomerase-based therapy in cancer.

\section{Discussion}

Early diagnosis and identification of new predictive and diagnostic biomarker has helped to determine the effectiveness of various therapies and the treatment response and predicting outcome of cancer treatment more accurately [26-28]. Overexpression of telomerase enzyme and consequential immortalization is a key step for cancer initiation and progression. Furthermore, telomerase has been shown to be necessary for maintaining tumor growth. Therefore, many inhibitors that suppress telomerase expression are currently under investigation for 


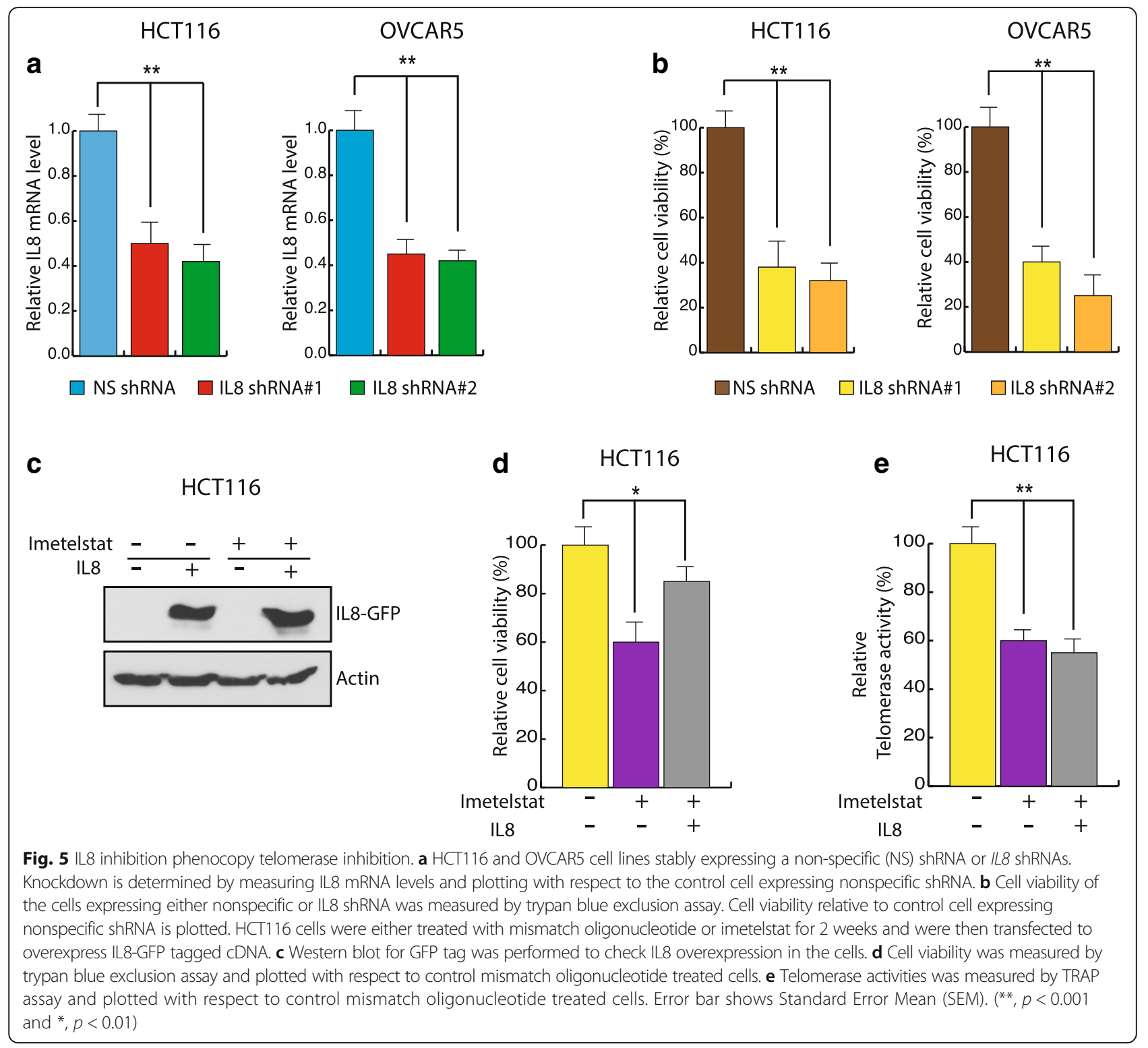

cancer treatment [29-31]. However, because of variability between the patient response to telomerase inhibition, identification of biomarkers that might predict cancers cell response to telomerase inhibitor will provide immense clinical benefits.

In our previous study, we showed that simultaneous inhibition of CDKN1A and telomerase by imetelstat leads to synergistic tumor growth inhibition [20]. In the current study, we have made an attempt to identify the biomarker that could predict telomerase inhibition response and to do that we performed gene expression microarray analysis on multiple ovarian and colon cancer cell lines that were responsive to telomerase inhibitor imetelstat treatment. Our results are summarized in Fig. 6 and discussed below.
The results shown in our study is more practical and advantageous because it's not based on hypothesis-based biomarker discovery. Our study is largely discovery-based biomarker identification, where we have employed unbiased high through-put based Transcriptome-wide gene expression analysis to discover a functional predictive biomarker of telomerase inhibition response. We have further employed secondary assays to validate and confirm our findings in multiple ovarian and colon cancer cell lines.

In our study, we show that different cell lines respond differently to telomerase inhibition. Next, we find that the cell lines that show growth inhibition phenotype upon telomerase inhibition, downregulate IL8 cytokine expression level. This phenomenon is of general 


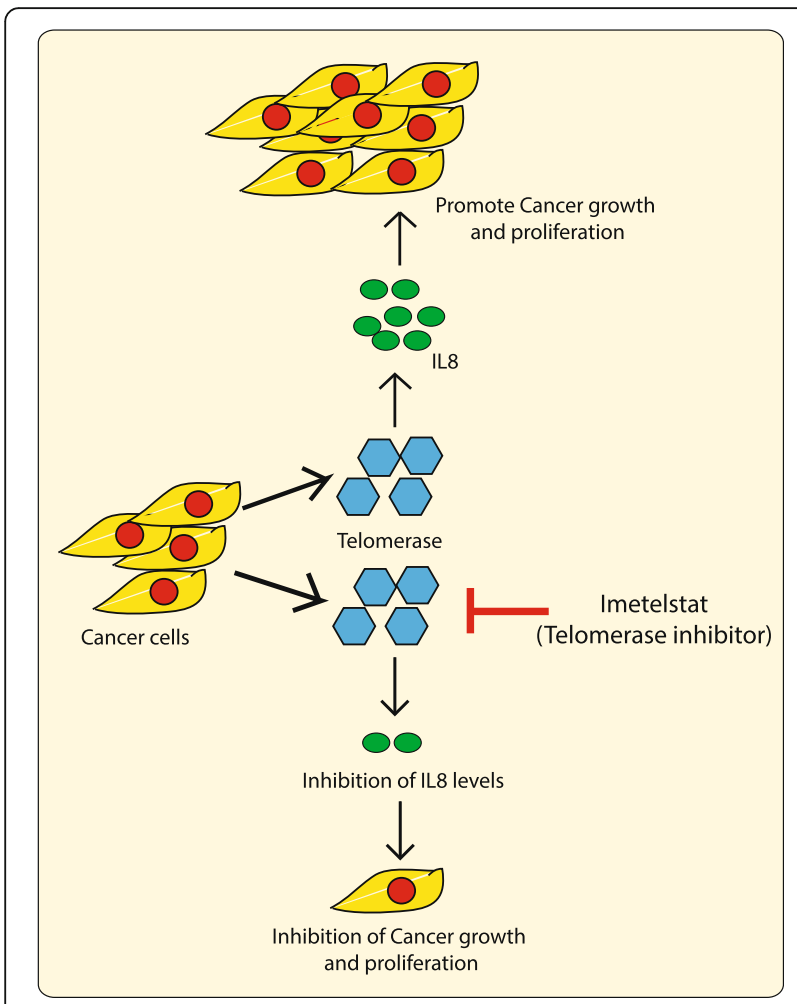

Fig. 6 IL8 is a biomarker that could predict telomerase inhibition response. Cancer cells exclusively produce enzyme telomerase which is now targeted with pharmacological inhibitors like imetelstat. Inhibition of Telomerase leads to inhibition of IL8, which is a pro-oncogenic cytokine and thus inhibits cancer cells growth and progression. IL 8 act as predictive biomarker for telomerase response occurrence as we find that multiple ovarian and colon cell lines show decrease in level of both IL8 mRNA and protein upon treatment with imetelstat. Additionally, we find that this phenomenon is specific for the cancer cell lines that show strong growth inhibition following imetelstat treatment along with concomitant decrease in telomerase expression. A previous study has shown that telomerase is bound to the promoters of a subset of NF- $\mathrm{kB}$ target genes, including IL6, IL8, and TNF- $\alpha$ and stimulate their expression to sustain inflammation and promote cancer progression [32]. These studies provide us an insight into possible mechanism by which inhibition of telomerase leads to decrease in IL8 levels. Based on the previous studies that document that telomerase is directly bound to IL8 promoter, in our studies we find that non-responder cancer cell lines which don't show significant decrease in telomerase level upon imetelstat treatment, also do not show any decrease in IL8 levels, again reconfirming that decrease in IL8 is dependent on telomerase inhibition. Therefore, this is a specific phenomenon observed only in cancer cell line that show telomerase inhibition induced growth suppression.
IL8 is a chemokine and is shown to be produced form macrophages and other type of cells [33]. Previous studies have shown that cancer cell over-express IL8 and its overexpression is associated with poor prognosis, increase in cell invasion that promotes cancer cell progression, angiogenesis, and metastases [34-36]. In fact, treatment based on inhibition of IL8 expression is shown to enhance the efficacy of the cancer-based therapies [37, 38]. Furthermore, our results show that inhibition of IL8 upon imetelstat treatment leads to inhibition of cell growth and proliferation, thus mimicking growth inhibition phenotype induced upon telomerase inhibition. In conclusion, our study identifies IL8 as an important biomarker that predict the effectiveness of telomerase-based therapy for treating cancer.

\section{Conclusion}

Our study employs gene expression analysis to identify a new biomarker that could predict the response to telomerase inhibition. This information can be utilized in clinical settings to determine whether patient will be responsive to telomerase-based therapy or not. There are many reliable methods that allows convenient and precise measurement of IL8 levels and hence it can be effectively utilized in clinics. Furthermore, future studies are needed to identify other such predictive biomarker that will facilitate to determine the effectiveness of various therapies and treatment response and predicting outcome of cancer treatment more accurately.

\section{Additional files}

Additional file 1: Figure S1. IC50 determination or Imetelstat. HCT116 and OVCAR5 cells were treated with various concentrations of Imetelstat. Cell viability was measured after $48 \mathrm{~h}$ of treatment by trypan blue exclusion assay. Error bar shows Standard Error Mean (SEM). ** represents $p<0.001$. (PDF 817 kb).

Additional file 2: Figure S2. Relative Telomere length measurement in multiple ovarian and cancer cell lines before and after Imetelstat treatment. HCT116, COLO205 and OVCAR5 cells were either treated with control mismatch oligo or Imetelstat for 2 weeks. Relative telomere length was measured using Relative Human Telomere length Quantification qPCR assay kit from Science cell. Error bar shows Standard Error Mean (SEM). * represents $p<0.01$. (PDF $242 \mathrm{~kb}$ ).

Additional file 3: Figure S3. IL8 level determination by immunoblotting in multiple ovarian and cancer cell lines before and after Imetelstat treatment. Cancer cells were either treated with control mismatch oligo or Imetelstat for 2 weeks. IL 8 protein level was measured by immunoblotting. Actin was used as loading control. (PDF $1171 \mathrm{~kb}$ ).

\section{Abbreviations}

CDKN1A: Cyclin dependent Kinase Inhibitor 1A; IL8: Interleukin 8;

TRAP: Telomeric Repeat Amplification Protocol

\section{Acknowledgements}

We would like to thank Yale pathology instrumentation core and Yale cancer center facility for providing us the equipment and resources.

\section{Availability of data and materials}

The datasets used and/or analyzed during the current study are available from the corresponding author on reasonable request. 


\section{Authors' contributions}

RG designed the experiments. PS, YD and RG performed the experiments. SD performed bioinformatics analysis. RG interpreted the data and wrote the manuscript. All authors have read and approved the final version of the manuscript.

\section{Ethics approval and consent to participate}

No ethical approval is required for using any of the cell lines employed for our study reported in this research article.

\section{Consent for publication}

Not applicable.

\section{Competing interests}

The authors declare that they have no competing interests.

\section{Publisher's Note}

Springer Nature remains neutral with regard to jurisdictional claims in published maps and institutional affiliations.

\section{Author details}

'Department of Pathology, Yale University School of Medicine, LH-306, New Haven, CT 06510, USA. ${ }^{2}$ Singapore Institute of Clinical Sciences, Agency for Science Technology and Research (A*STAR), Brenner Centre for Molecular Medicine, 30 Medical Dr., Singapore 117609, Singapore.

Received: 15 December 2017 Accepted: 25 June 2018

Published online: 09 July 2018

\section{References}

1. Cheng JF, Smith CL, Cantor CR. Isolation and characterization of a human telomere. Nucleic Acids Res. 1989;17(15):6109-27.

2. DeLange AM, McFadden $\mathrm{G}$. Efficient resolution of replicated poxvirus telomeres to native hairpin structures requires two inverted symmetrical copies of a core target DNA sequence. J Virol. 1987:61(6):1957-63.

3. Webb CJ, Wu Y, Zakian VA. DNA repair at telomeres: keeping the ends intact. Cold Spring Harb Perspect Biol. 2013;5(6)

4. de Lange T. How telomeres solve the end-protection problem. Science. 2009;326(5955):948-52

5. Sfeir A, de Lange T. Removal of shelterin reveals the telomere endprotection problem. Science. 2012;336(6081):593-7.

6. Arnoult N, Karlseder J. Complex interactions between the DNA-damage response and mammalian telomeres. Nat Struct Mol Biol. 2015;22(11): 859-66.

7. Osterhage $\mathrm{LL}$, Friedman KL. Chromosome end maintenance by telomerase J Biol Chem. 2009;284(24):16061-5.

8. Cong YS, Wright WE, Shay JW. Human telomerase and its regulation. Microbiol Mol Biol Rev. 2002;66(3):407-25. table of contents

9. Martinez P, Blasco MA. Replicating through telomeres: a means to an end. Trends Biochem Sci. 2015:40(9):504-15.

10. Hahn WC, Meyerson M. Telomerase activationcellular immortalization and cancer. Ann Med. 2001;33(2):123-9.

11. Shay JW, Wright WE. Senescence and immortalization: role of telomeres and telomerase. Carcinogenesis. 2005;26(5):867-74.

12. Chiba K, Johnson JZ, Vogan JM, Wagner T, Boyle JM, Hockemeyer D. Cancer-associated TERT promoter mutations abrogate telomerase silencing. Elife. 2015:4

13. Holysz H, Lipinska N, Paszel-Jaworska A, Rubis B. Telomerase as a useful target in cancer fighting-the breast cancer case. Tumour Biol. 2013;34(3): 1371-80.

14. Shay JW, Keith WN. Targeting telomerase for cancer therapeutics. Br J Cancer. 2008;98(4):677-83.

15. Shay JW, Wright WE. Telomerase: a target for cancer therapeutics. Cancer Cell. 2002;2(4):257-65.

16. Ivancich M, Schrank Z, Wojdyla L, Leviskas B, Kuckovic A, Sanjali A, Puri N. Treating Cancer by targeting telomeres and telomerase. Antioxidants (Basel). 2017;6(1)

17. Chiappori AA, Kolevska T, Spigel DR, Hager S, Rarick M, Gadgeel S, Blais N, Von Pawel J, Hart L, Reck M, et al. A randomized phase II study of the telomerase inhibitor imetelstat as maintenance therapy for advanced nonsmall-cell lung cancer. Ann Oncol. 2015;26(2):354-62.
18. Damm K, Hemmann U, Garin-Chesa P, Hauel N, Kauffmann I, Priepke H, Niestroj C, Daiber C, Enenkel B, Guilliard B, et al. A highly selective telomerase inhibitor limiting human cancer cell proliferation. EMBO J. 2001; 20(24):6958-68.

19. Xu Y, Goldkorn A. Telomere and telomerase therapeutics in Cancer. Genes (Basel). 2016;7(6)

20. Gupta R, Dong Y, Solomon PD, Wettersten HI, Cheng CJ, Min JN, Henson J, Dogra SK, Hwang SH, Hammock BD, et al. Synergistic tumor suppression by combined inhibition of telomerase and CDKN1A. Proc Natl Acad Sci U S A. 2014;111(30):E3062-71.

21. Roth A, Harley CB, Baerlocher GM. Imetelstat (GRN163L)-telomerase-based cancer therapy. Recent Results Cancer Res. 2010;184:221-34.

22. Burchett KM, Yan Y, Ouellette MM. Telomerase inhibitor Imetelstat (GRN163L) limits the lifespan of human pancreatic cancer cells. PLoS One. 2014;9(1):e85155.

23. Wege H, Chui MS, Le HT, Tran JM, Zern MA. SYBR green real-time telomeric repeat amplification protocol for the rapid quantification of telomerase activity. Nucleic Acids Res. 2003;31(2):E3.

24. Harley CB. Telomerase and cancer therapeutics. Nat Rev Cancer. 2008;8(3): 167-79.

25. Shammas MA, Koley H, Bertheau RC, Neri P, Fulciniti M, Tassone P, Blotta S, Protopopov A, Mitsiades C, Batchu RB, et al. Telomerase inhibitor GRN163L inhibits myeloma cell growth in vitro and in vivo. Leukemia. 2008;22(7): $1410-8$.

26. Mehta S, Shelling A, Muthukaruppan A, Lasham A, Blenkiron C, Laking G, Print C. Predictive and prognostic molecular markers for cancer medicine. Ther Adv Med Oncol. 2010;2(2):125-48.

27. Buonaguro FM, Pauza D, Tornesello ML, Hainaut P, Franco R, Marincola FM. Cancer diagnostic and predictive biomarkers. Biomed Res Int. 2014;2014 980163.

28. Kalia M. Biomarkers for personalized oncology: recent advances and future challenges. Metabolism. 2015;64(3 Suppl 1):S16-21.

29. Hanahan D, Weinberg RA. Hallmarks of cancer: the next generation. Cell. 2011;144(5):646-74.

30. Gomez DL, Armando RG, Cerrudo CS, Ghiringhelli PD, Gomez DE. Telomerase as a Cancer target development of new molecules. Curr Top Med Chem. 2016:16(22):2432-40.

31. Jager K, Walter M. Therapeutic targeting of telomerase. Genes (Basel). 2016;7(7)

32. Ghosh A, Saginc G, Leow SC, Khattar E, Shin EM, Yan TD, Wong M, Zhang Z, Li G, Sung WK, et al. Telomerase directly regulates NF-kappaB-dependent transcription. Nat Cell Biol. 2012;14(12):1270-81.

33. Arango Duque G, Descoteaux A. Macrophage cytokines: involvement in immunity and infectious diseases. Front Immunol. 2014:5:491.

34. Wang Y, Xu RC, Zhang XL, Niu XL, Qu Y, Li LZ, Meng XY. Interleukin-8 secretion by ovarian cancer cells increases anchorage-independent growth, proliferation, angiogenic potential adhesion and invasion. Cytokine. 2012; 59(1):145-55.

35. Kuai WX, Wang Q, Yang XZ, Zhao Y, Yu R, Tang XJ. Interleukin-8 associates with adhesion, migration, invasion and chemosensitivity of human gastric cancer cells. World J Gastroenterol. 2012;18(9):979-85.

36. Todorovic-Rakovic N, Milovanovic J. Interleukin-8 in breast cancer progression. J Interf Cytokine Res. 2013;33(10):563-70.

37. Singh JK, Simoes BM, Howell SJ, Farnie G, Clarke RB. Recent advances reveal IL-8 signaling as a potential key to targeting breast cancer stem cells. Breast Cancer Res. 2013;15(4):210

38. Juvekar A, Wulf GM. Closing escape routes: inhibition of IL-8 signaling enhances the anti-tumor efficacy of PI3K inhibitors. Breast Cancer Res. 2013; 15(2):308. 